\title{
Thermal conditions of load-bearing elements of radioactive waste storage facilities
}

\author{
P.V. Yakovlev ${ }^{1, *}$, V.A. Lebedev ${ }^{1}$, and V.M. Piskunov ${ }^{1}$ \\ ${ }^{1}$ Saint-Petersburg Mining University, 2, 21st Line, St Petersburg 199106, Russia
}

\begin{abstract}
Facilities for storing radioactive waste with residual heat differ from other facilities in high safety standards in all operating conditions. A feature of these structures is the presence of biological protection in the form of walls and ceilings made of reinforced concrete with a large thickness. The combination of heat dissipation and the large thickness of walls and floors create conditions for the appearance of significant thermal stresses. This feature should be taken into account in the strength calculations of these buildings, taking into account the summation of seismic effects, gravitational forces and thermal stresses caused by uneven temperature fields in concrete. The paper presents the results of calculations of the combined effects of thermal stresses and earthquakes on a building. The data obtained made it possible to determine the features of the deformation of concrete structures with a combination of loads.
\end{abstract}

\section{Introduction}

A significant amount of nuclear fuel waste has already been accumulated. This explains the relevance of the study of storage and transport facilities for spent fuel elements. One of the ways to store waste is to create specialized facilities capable of storing them at a safe level.

Radioactive waste storage buildings are designed for a combination of loads, including operational, emergency and earthquakes. Additional loads arising during earthquakes are taken into account in the design model in addition to the gravitational acceleration forces described by a three-component accelerogram. Among the features of the operating mode of spent nuclear fuel storage are significant heat release, which is removed to the environment by cooling systems. Of the existing heat removal systems, the air cooling system is considered safer, since cooling is carried out due to the free convection of atmospheric air with its removal into the environment. The lack of air as a heat carrier should be attributed to the low density and heat capacity of the air, which results in zones of local overheating of fuel elements and storage structures. [1-9] The influence of temperature fields on the strength of the structure in the system of standard combinations of loads should be taken into account [10]. The computational scheme of the model of a "dry" storage for spent fuel elements is shown in Fig. 1.

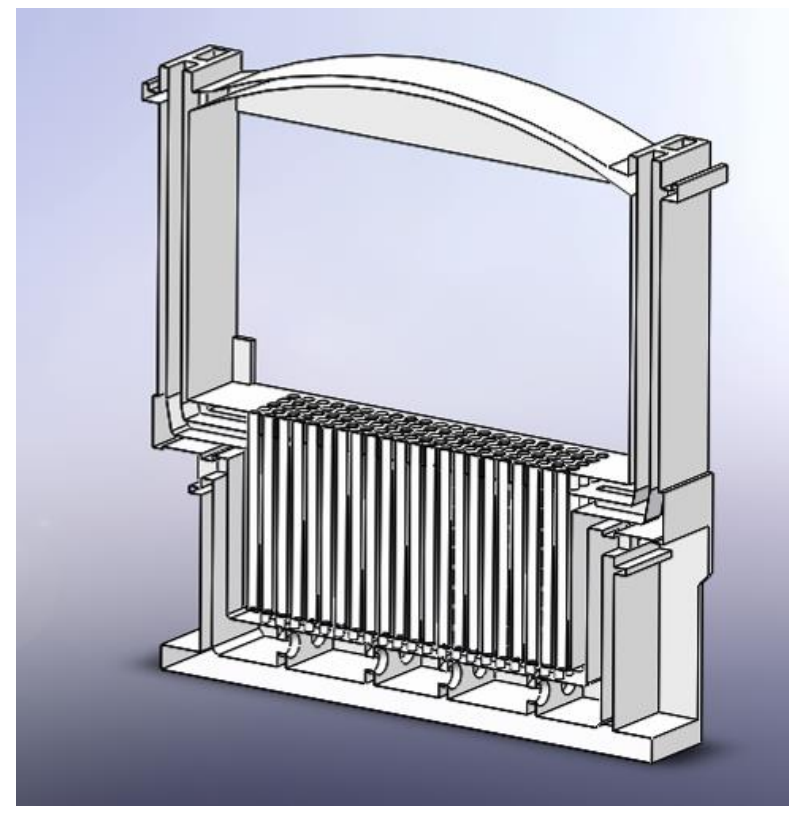

Fig. 1. Calculation diagram of the model of the "dry" port storage of spent fuel elements.

\section{Methods and Materials}

The object has a complex geometric shape and consists of elements with different thermophysical and strength properties. Also, different boundary conditions must be used for different surfaces. In this regard, the method of numerical modeling was chosen for the study. To determine the deformations and stresses in the bearing elements, the problems of modeling free convective heat

Corresponding author: yakovlev pv@pers.spmi.ru 
transfer on surfaces and the problem of energy transfer by thermal conductivity in solids (concrete structures) are solved.

Single-phase homogeneous cooling air fluid in dry storage is characterized by:

1. temperature field $T\left(x_{i}, \tau\right)$ - scalar field;

$$
\stackrel{2 .}{W}\left(x_{i}, \tau\right)=\stackrel{\text { field }}{\stackrel{i}{i}}+W_{y} \stackrel{j}{j}+W_{z} \stackrel{\text { of }}{k}-
$$

speed

field;

3. pressure field $P\left(x_{i}, \tau\right)$ - scalar field,

Where: $\mathrm{W}$ - free convective speed, $\mathrm{m} / \mathrm{s} ; \mathrm{P}-$ pressure, $\mathrm{Pa} ; \mathrm{x}_{\mathrm{i}}-$ orthogonal coordinate system, $\mathrm{m} ; \tau-$ time, $s$.

To calculate temperature, pressure and, in general, the three components of the velocity vector, it is necessary to solve five differential equations:

- differential equation of energy transfer in a fluid the Fourier-Kirchhoff equation;

- three differential equations of momentum transfer in a fluid medium - Navier - Stokes equations;

- differential equation of continuity [11-14].

Fourier-Kirchhoff differential equation

In vector form, the equation of energy transfer in a fluid medium has the form [15-16]:

$$
\rho * c\left(\frac{\partial T}{\partial \tau}+\vec{W} * \nabla T\right)=\operatorname{div}[\lambda * \operatorname{grad}(T)+\mu * \Phi-\mathrm{P} * \operatorname{div} \overrightarrow{\mathrm{W}}]
$$

Where $\rho^{*} c * \partial T / \partial \tau-$ term on the right side of the energy equation, which reflects the nonstationarity of the heat transfer process; $\rho * c * \vec{W} * \nabla T$ convective term of the energy equation - takes into account the transfer of heat due to the movement of the medium; $\operatorname{div}[\lambda * \operatorname{grad}(T)]-$ diffusion term of the equation - takes into account the transfer of heat by thermal conductivity; $\mu * \Phi-$ term in the energy equation that takes into account the heating of the medium due to the dissipation of the kinetic energy of motion due to friction; $\mu$ - dynamic viscosity index; $\Phi-$ dissipative function; $-P * \operatorname{div} \vec{W}-$ a term in the equation that takes into account the change in the energy of the fluid during its compression or expansion.

Taking the assumption that the physical properties of the medium are independent of temperature and that there are no internal sources of heat, the FourierKirchhoff equation takes the form [17-21]:

$$
\rho * c\left(\frac{\partial T}{\partial \tau}+\vec{W} * \nabla T\right)=\operatorname{div}[\lambda * \operatorname{grad}(T)]
$$

To solve the Fourier-Kirchhoff equation, it is necessary to first calculate the velocity field by solving the Navier-Stokes equations.

The Navier Stokes equation for fluids with constant density in vector form has the form $[10,11]$ :

$$
\frac{\partial \mathscr{W}}{\partial \tau}+\stackrel{W}{W} * \nabla \mathscr{W}=g-\frac{1}{\rho} \nabla P+v * \nabla^{2} \mathscr{W}
$$

In this case, the continuity equation takes the form:

$$
\operatorname{div} \tilde{W}=0
$$

The uniqueness conditions necessary for solving the system of differential equations of convective heat transfer are defined in [22-27].

- To highlight the only solution, the unambiguous conditions were set:

- the geometry of the computational domain and its dimensions, for which a three-dimensional computational dynamic model of the dry storage was used;

- boundary conditions for calculating the velocity field: equality to zero of the air velocity on solid surfaces (adhesion condition) and atmospheric pressure at the inlet and outlet of the ventilation system;

- boundary conditions for calculating heat transfer: boundary conditions of the second kind on the surface of canisters and boundary conditions of the third kind on the rest of the surfaces of contact of air with solid surfaces;

- C. ${ }^{\circ}$ ambient temperature is assumed to be $20^{\circ} \mathrm{C}$

\section{Results}

The results of calculating the velocity field are shown in Fig. 2, temperature fields in Fig. 3.

Shown in Fig. 2, the velocity field, we see that the air velocity around the cylinders is the same in all parts of the building. This result is obtained due to the low resistance of the distribution part of the air channel and the high resistance near the top of the storage area. The expected result should be equalization of the temperature field, but the result was not achieved. In fig. 3 shows the temperature field, where it can be seen that the cooling air has a high temperature in the upper part of the storage area. This is due to the influence of two factors: 1 . The influence of gravitational forces. The heated air rises. 2. Air heated in the central part of the storage area moves horizontally and is additionally heated in contact with heated elements. 


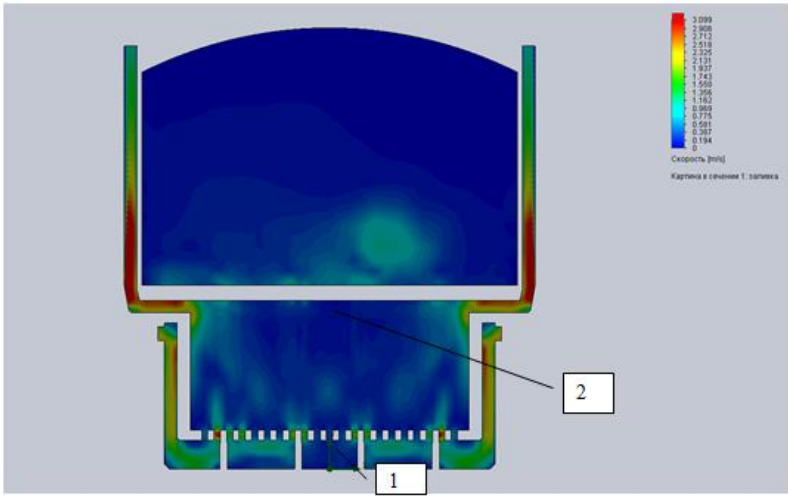

Fig. 2. Estimated speed $(\mathrm{m} / \mathrm{s})$ of the air flow in the cooling air ducts of the radioactive waste storage zone in the storage slots; 1 - zone of "stagnation" of the air flow in the air ducts; 2 - zone of "stagnation" of air flow in the storage.

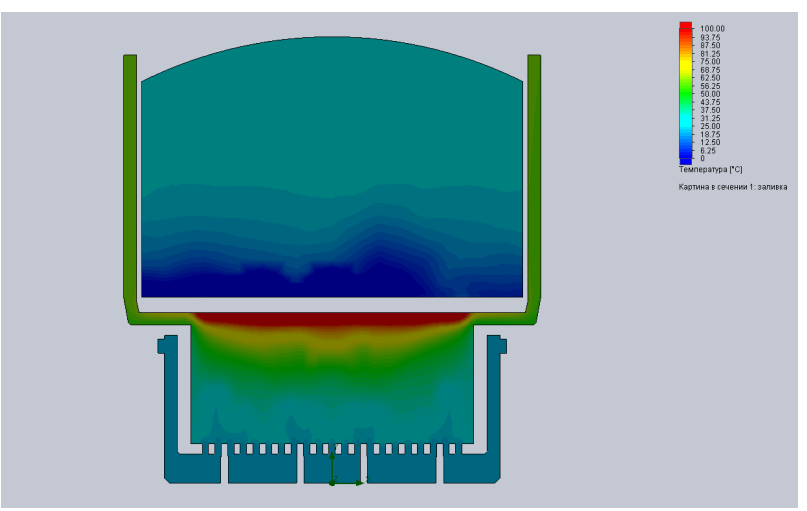

Fig. 3. Calculated temperature field $\left({ }^{\circ} \mathrm{C}\right)$ and temperature load on the building structures of the radioactive waste storage.

The calculated values of temperatures are shown in Figure 4. The calculated values of stresses, displacement and deformation of the slab due to thermal effects are shown in Fig. 5 - 8.

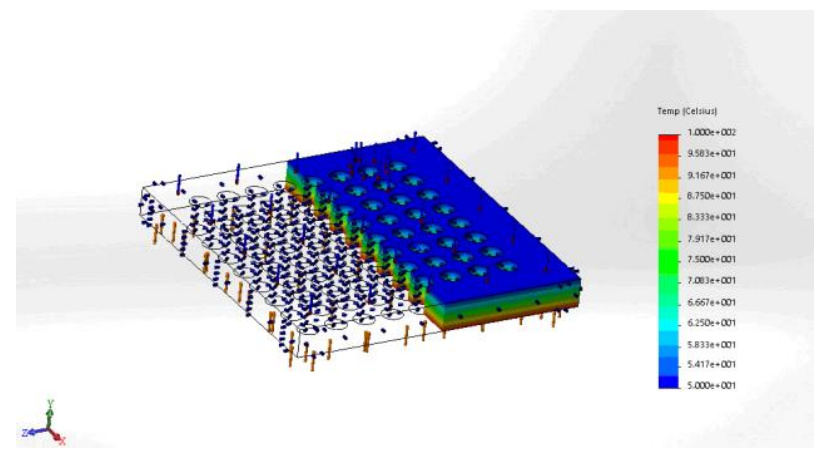

Fig. 4. Calculated temperature field of the storage plate (in section), ${ }^{\circ} \mathrm{C}$.

Heating a concrete slab from the bottom side and cooling it in the upper part leads to significant unevenness of the temperature field in the slab, as shown in Fig. 4.

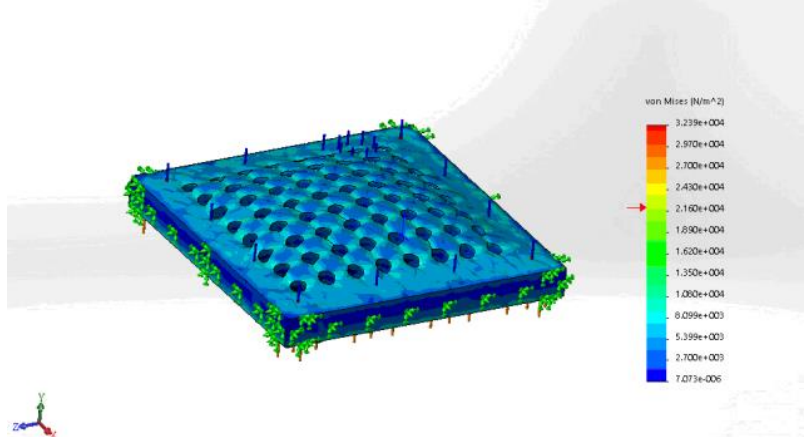

Fig. 5. Design stress in the storage slab due to thermal effects on the slab, Pa.

The unevenness of the temperature field in the slab, combined with its significant thickness, leads to the appearance of thermal stresses (Fig. 5). A special feature of concrete is its ability to resist compression and low tensile strength. As a result of these features, the plate is deformed upward (Fig. 6).

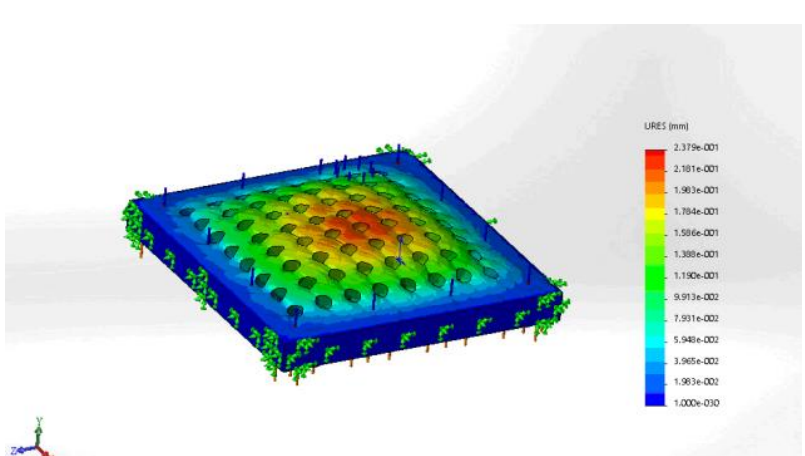

Fig. 6. Calculated deformations of the storage slab due to thermal effects on the slab, $\mathrm{mm}$.

The results of calculating the maximum deformations of the supporting structures are shown in Fig. 7.

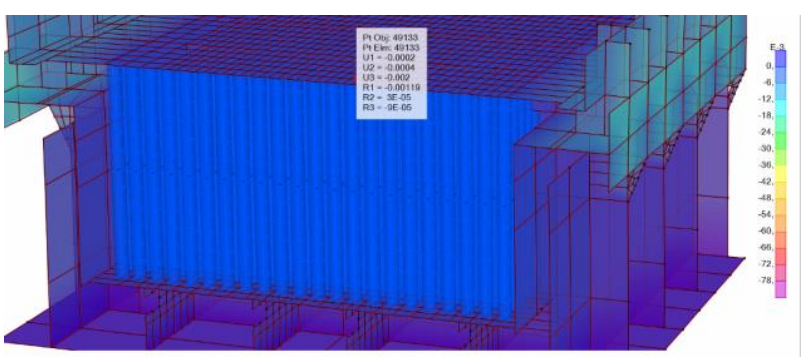

Fig. 7. Deformations of building structures as a result of seismic impact, $\mathrm{m}$.

The results obtained were used to study the combined effect of thermal stresses and stresses arising from an earthquake. The results of calculating the deformation of a building during an earthquake are shown in Fig. 7. Zones of maximum deformations during seismic impacts are not observed in places affected by the concentration of thermal stresses. 


\section{Discussion}

An analysis of the temperature fields, stresses and deformations of the slab shows that overheating of the lower part of the slab with a thickness of $1100 \mathrm{~mm}$ leads to significant thermal stresses, comparable in magnitude with the limiting ones for B40 concrete. As seen from Fig. 6, thermal stresses lead to noticeable deformations of the storage slab, up to $2 \mathrm{~mm}$ or more for every $10 \mathrm{~m}$ of the slab length. In addition, deformations of the bottom of the slab can lead to pinching of the plugs.

A feature of the deformation of a reinforced concrete slab should be noted its deformation upward, towards the region of low temperatures. This is due to the peculiarities of the mechanical properties of concrete, which perceives high compressive loads and are almost 10 times less in tension.

\section{Conclusion}

The computational analysis of the deformations of the nuclear waste storage slab determines the need for additional study of the temperature fields and thermal stresses arising in the load-bearing elements of the port terminal and their effect on the integrity and loadbearing capacity of the building elements. The problem of the occurrence of thermal stresses implies the need to develop new structural solutions for buildings, to reduce operational loads to increase their reliability and safety.

\section{References}

[1] V.G. Fetisov, A.K. Nikolaev, Y.V. Lykov, Aggregative simulation method for implementing mathematical models for gas transmission systems, The IOP Conference Series: Materials Science and Engineering 327, 022033(2018)

[2] V.G. Fetisov, A.K. Nikolaev, Y.V. Lykov, Experimental Studies for Determining Gas Flow Rate Accidental Release on Linear Part of Pipeline, IOP Conference Series: Earth and Environmental Science, 1-6 (2017)

[3] F.M. Arabloo, A.H. Mohammadi, Efficient estimation of natural gas compressibility factor using a rigorous method, Journal of natural gas science and engineering 4, 8-17 (2014)

[4] V.G. Fetisov, A.K. Nikolaev, Y.V. Lykov, L.N. Duchnevich, Mathematical modeling of nonstationary gas flow in gas pipeline, The IOP Conference Series: Materials Science and Engineering 327, 022034 (2018)

[5] A.M. Jaeschke, Accurate prediction of compressibility factors by the GERG virial equation, SPE Production engineering 6, 343-349 (1991)

[6] J. Modisette, Instability and other numerical problems in finite difference pipeline models, PSIG Annual meeting New Mexico 5, 22-27 (2012)
[7] N.N. Panasenko, P.V. Yakovlev, M.F. Rudenko, N.V. Selivanov, V.G. Bukin, Strength of Carrier Structures of Maritime Ships and Port Facilities Under the Impact of Lightning, International Conference on Modern Trends in Manufacturing Technologies and Equipment 2018 11, 1, 580-585 (2019)

[8] M. Chaczykowski, Sensitivity of pipeline gas flow model to the selection of the equation of state, Chemical engineering research and design 3, 159163 (2009)

[9] F. Dauber, R. Span, Achieving higher accuracies for process simulations by implementing the new reference equation for natural gases, Computers and chemical engineering 7, 15-21 (2012)

[10] A.A. Abouellail, M.A. Kostina, S.I. Bortalevich, E.L. Loginov, Y.A. Shinyakov, M.P. Sukhorukov, Building Information Model: advantages, tools and adoption efficiency, The IOP Conference Series: Materials Science and Engineering 327, 022002 (2018)

[11] A.A. Tarasenko, P.V. Chepur, A.A. Gruchenkova, 2018 Analysis of stress-strain state of RVS-20000 tank under non-axisymmetric wind load action, The IOP Conference Series: Materials Science and Engineering 327, 022039 (2018)

[12] SP 16.13330.2011 2011, Steel Construction (Moscow, The Ministry of regional development of the Russian Federation) 177

[13] V.A. Bruyaka, E.A. Fokin, E.A. Soldusova, N.A. Glazunova, I.E. Adeyanov, Engineering analysis in ANSYS Workbench (Samara: Samara state technical University, 2010) 271

[14] G.E. Korobkov, R.M. Zaripov, I.A. Shammazov, Numerical modeling of the stress-strain state and stability of pipelines and tanks in complicated operating conditions (Saint-Petersburg: Nedra, 2009) 410

[15] A.A. Tarasenko, P.V. Chepur, A. Gruchenkova, MATEC Web of Conferences 73, 01025 (2016)

[16] A.A. Tarasenko, P.V. Chepur, Soil Mechanics and Foundation Eng. 53, 238-243 (2016)

[17] H. Karagülle, L. Malgaca, H. Öktem, Smart Materials and Structures 13, 661-667 (2004)

[18] A.I. Orlenko, N.K. Kuznetsov, Q.T. Vuong, Devices for the transformation of motion in the structure of oscillatory system: dynamic damping of oscillations, The IOP Conference Series: Materials Science and Engineering 327, 022078 (2018)

[19] S.V. Eliseev, A.I. Artyunin, Applied theory of oscillations in problems of dynamics of linear mechanical systems (Novosibirsk, Nauka, 2016) 459

[20] I.A. Karnovsky, E. Lebed, Theory of vibration protection (Switzerland, Springer, 2016) 708

[21] S.V. Belokobyiski, S.V. Eliseev, V.I. Kashuba, Applied problems of the structural theory of 
vibrtaion protection systems (Saint-Petersburg, Polytechnic, 2013) 363

[22] S.V. Eliseev, A.P. Khomenko, Dynamic vibration damping: the feedback concept and structural methods of mathematical modeling (Novosibirsk, Nauka, 2014) 357

[23] S.V. Eliseev, A.I. Orlenko, D.H. Nguyen, Devices for transformation of motion in the structure of a dyad of a mechanical oscillatory system, Proceeding of the Don State Technical University 3, 46-59 (2017)

[24] V.V. Dezhin, On damping of screw dislocation bending vibrations in dissipative crystal: limiting cases, The IOP Conference Series: Materials Science and Engineering 327, 032017 (2018)

[25] N.N. Panasenko, P.V. Yakovlev, M.F. Rudenko, N.V. Selivanov, V.G. Bukin, Strength of Carrier Structures of Maritime Ships and Port Facilities Under the Impact of Lightning, International Conference on Modern Trends in Manufacturing Technologies and Equipment 2018 11, 1, 580-585 (2019)

[26] A.D. Gorynin, V.Yu. Antsev, A.N. Shaforost, Dynamic loads during failure risk assessment of bridge crane structures, The IOP Conference Series: Materials Science and Engineering 327, 042040 (2018)

[27] V.A. Gulevsky, A.N. Belyaev, T.V. Trishina, Torsional vibrations of shafts of mechanical systems, The IOP Conference Series: Materials Science and Engineering 327, 042041 (2018)

[28] I.V. Kudinov, V.A. Kudinov, A.V. Eremin, V.V. Zhukov, Mathematical model of rod oscillations with account of material relaxation behaviour, The IOP Conference Series: Materials Science and Engineering 327, 042059 (2018) 